\title{
Activity patterns and their relationship to the habitat use of mule deer (Odocoileus hemionus) in the Chihuahuan Desert, Mexico
}

\author{
Luz Adriana Pérez-Solano ${ }^{1 *}$ y Sonia Gallina Tessaro' \\ ${ }^{1}$ Red de Biología y Conservación de Vertebrados, Instituto de Ecología A. C. Carretera Antigua a Coatepec 351, El Haya CP. 91070, \\ Xalapa. Veracruz, México. Email: adriana.perez.s@outlook.com (LAP-S), sonia.gallina@inecol.mx (SGT). \\ "Corresponding author
}

In desert regions, species have adapted physiologically and behaviorally to the extreme conditions of their environment, such as a lack of water and extreme temperatures. This is the case of the mule deer (Odocoileus hemionus) a species which many aspects of its relationship between behavioral ecology and habitat characteristics are unknown. The objectives of this study were: 1) to characterize the daily activity pattern of female mule deer in relationship to three behavioral patterns: resting, feeding and moving, and 2) assess the relationship between these behavioral patterns and the characteristics of the habitat. This study was conducted in the Chihuahuan Desert, where six females of mule deer were monitored using VHF radiotelemetry between 2012 and 2014 . We distinguish the behavioral patterns by sensors in the collar and we quantified the number of beeps emitted per minute. Moreover, we described the daily pattern associated with these activities using Kernel density graphs and evaluated the effect of habitat variables on the frequency of resting and feeding behaviors applying a generalized linear model (GLM). We identified the location in which feeding and resting behaviors occurred, and the type of plant associations present and the percentage of area they occupied. We recorded locations over 328 days/deer. Female mule deer spent most of their time resting (63.41 \%), followed by moving ( $21.26 \%)$, and feeding (15.34 \%). Deer movement and feeding behaviors were most frequent during the crepuscule. The temperature was the only variable that influenced resting and feeding behaviors (deviance $=4.7 ; d . f .=1 ; P=0.02$ ). The estimated area used for resting was $10.35 \mathrm{~km}^{2}$, while the corresponding area for feeding was $6.45 \mathrm{~km}^{2}$, in which the plant association with dominance of Larrea tridentata, Opuntia rastrera and Fouquieria splendens was the most common. Considering the high temperatures prevailing on the region, resting most of the day likely helps deer to have an adequate thermoregulation. The time dedicated to feeding is less than the dedicated to other activities; deer may be spending more time moving than feeding due to the spatial distribution of suitable food patches, or to avoid predators. The mule deer occupy the same areas to feed and rest; however, feeding areas are smaller those used to rest, these areas have been identified in previous studies as those with the greatest use (core areas). In the plant association with greater occupation within the feeding zones, there are species reported as an essential for the deer diet; these areas are located at the foot of the hill, which gives the deer thermal protection.

En regiones desérticas las especies se han adaptado fisiológica y conductualmente a la falta de agua, así como a las temperaturas extremas. Tal es el caso del venado bura (Odocoileus hemionus), especie de la cual muchos aspectos de su ecología conductual y su relación con el hábitat aún son desconocidos. Los objetivos de este estudio fueron: 1) caracterizar el patrón diario de actividad de hembras de venado bura con relación a tres pautas de comportamiento: descanso, alimentación y movimiento, y 2) evaluar la relación entre estas pautas y las características del hábitat. Este estudio se realizó en el Desierto Chihuahuense, donde se monitorearon mediante radiotelemetría VHF seis hembras de venado bura entre el 2012 y el 2014. Las pautas se distinguieron mediante un sensor en el collar y la cuantificación del número de pulsaciones que emite por minuto. Se caracterizó el patrón de actividad asociado con las pautas utilizando gráficos de densidad Kernel y se evaluó el efecto de variables del hábitat sobre la frecuencia de las mismas aplicando un modelo lineal generalizado (GLM). Se identificaron las áreas que las venadas usan para alimentarse y descansar, así como las asociaciones vegetales presentes en esas áreas y el porcentaje que ocupaban. Se registraron localizaciones durante un total de 328 días/venado. Las hembras de venado bura dedican la mayor parte del tiempo a descansar (63.41 \%), seguido de realizar movimientos (21.26 \%) y por último a alimentarse (15.34\%). Tienen horarios crepusculares de movimiento y alimentación. La temperatura fue la única variable que afectó a las pautas de descanso y alimentación (devianza $=4.7 ; g . l .=1 ; P=0.02$ ). El área estimada para las zonas que seleccionan para descansar fue de $10.35 \mathrm{~km}^{2}$ y la de alimentación fue de $6.45 \mathrm{~km}^{2}$, donde predominó la asociación vegetal con dominancia de Larrea tridentata, Opuntia rastrera y Fouquieria splendens. Considerando las altas temperaturas que predominan en el sitio, el permanecer echadas la mayor parte del día puede ser necesario para una adecuada termorregulación. El tiempo dedicado a alimentarse es menor al de las otras pautas, esto podría indicar que, las venadas están invirtiendo mayor tiempo en desplazarse que en alimentarse debido a la distribución espacial de parches adecuados para su alimentación, o para evadir depredadores. Las venadas ocupan los mismos sitios para alimentarse y descansar, sin embargo, las áreas para lo primero son menores, dichas áreas han sido identificadas en trabajos previos como las zonas de las áreas núcleo de actividad. En la asociación vegetal con mayor ocupación dentro de las zonas de alimentación se encuentran especies reportadas como parte esencial de la dieta del venado, ubicadas al pie del cerro, lo cual confiere a los venados protección térmica.

Key words: arid ecosystems; behavior patterns; cervid; Mapimí; radiotelemetry.

(c) 2019 Asociación Mexicana de Mastozoología, www.mastozoologiamexicana.org

\section{Introducción}

El estudio de la ecología conductual de la fauna busca describir el comportamiento de los animales en función de la adaptación a las condiciones ecológicas, incluyendo tanto el entorno físico como al entorno ecológico (competidores, depredadores, etc.; Davies et al. 2012). Las respuestas conductuales de los individuos dependen de las condiciones de su hábitat (Gallina y Bello 2002) y pueden llegar a ser muy complejas y diferentes, tanto entre individuos como dependiendo del sexo y edad (Marchinton y Hirth 1984; Gallina y Bello 2014). 
El venado bura (Odocoileus hemionus) se distribuye principalmente en zonas templadas y áridas, desde el sur de Alaska hasta el norte de México (Anderson y Wallmo 1984; Geist 1998), donde se extiende por los estados de Baja California, Sonora, Chihuahua, Coahuila y Durango (Heffelfinger et al. 2006). En las regiones áridas y semiáridas de su distribución, la especie se ha adaptado morfológica y conductualmente a las condiciones extremas del desierto como la falta de agua y las temperaturas extremas. La actividad del venado bura ha sido asociada con la presencia de pendientes pronunciadas y terrenos accidentados (que además facilitan su escape ante los depredadores) o la existencia de cobertura vegetal de altura media, estos rasgos del paisaje le permiten tener una mejor protección térmica. De igual modo, los venados bura tienden a modificar sus movimientos y ámbitos hogareños a través del día y temporadas del año para hacer más eficiente el uso de los recursos (Sánchez-Rojas y Gallina 2000; Avey et al. 2003; Marshal et al. 2006; Pérez-Solano et al. 2016; 2017). Al interior de los ámbitos hogareños de los venados se presentan zonas con mayor densidad de recursos críticos, que son, por lo tanto, más importantes para su actividad (Burt 1943; Powell 2000). Conocer estas áreas de uso de los venados bura ofrece un importante marco de referencia para definir las características de los sitios seleccionados para su refugio y la obtención de recursos alimenticios seguros, además de contribuir a entender la ecología de su comportamiento (Burt 1943; Kaufman 1962; Samuel y Green 1988).

En México, muchos aspectos biológicos y ecológicos sobre el venado bura, necesarios para su manejo, son desconocidos, o la información es muy escasa, por lo que se utiliza información de estudios realizados en Estados Unidos (Pérez-Solano et al. 2016). En comparación con el venado cola blanca (O. virginianus), los trabajos de investigación que abordan aspectos conductuales del venado bura son muy pocos, por ejemplo, la conducta en relación a su ecología espacial en el desierto de Sonora (Alcalá-Galván y Krausman 2013) y en el Desierto Chihuahuense (Pérez-Solano et al. 2016; 2017). De acuerdo con la última revisión de estudios de cérvidos en México, únicamente un $13 \%$ de estos estudios incluyen a O. hemionus, y se enfocan principalmente en sus hábitos alimentarios, dinámica y densidades poblacionales, uso de hábitat y posibles reintroducciones en ciertas áreas (Mandujano 2004). Por lo tanto, resulta altamente relevante el estudio de aspectos conductuales de esta especie para comprender su ecología, mantener sus poblaciones y contar con información que contribuya a definir estrategias de conservación y mejores programas de aprovechamiento de este cérvido. Los objetivos de este estudio fueron: 1) caracterizar el patrón diario de actividad de hembras de venado bura con relación a tres pautas de comportamiento: descanso, alimentación y movimiento; y 2) evaluar la relación entre estas pautas de comportamiento y las características del hábitat.

\section{Material y métodos}

Área de estudio. El estudio se realizó en la región central del Desierto Chihuahuense, en la Reserva de la Biosfera de Mapimí (RBM), México, ubicada en la intersección de los estados de Durango, Chihuahua y Coahuila (CONANP 2006). Al centro de la RBM se localiza el cerro San Ignacio $\left(26^{\circ} 42^{\prime}\right.$, $26^{\circ} 38^{\prime} \mathrm{N},-103^{\circ} 42^{\prime},-103^{\circ} 48^{\prime} \mathrm{W}$; Figura 1), alrededor del cual se realizó este estudio. La vegetación dominante son los matorrales xerófilos, micrófilos y chaparrales de distintas composiciones, con vegetación halófita en las planicies (CONANP 2006; Rzedowski 2006). El clima es seco y extremoso semi-cálido con lluvias en verano, con una precipitación promedio anual de $271 \mathrm{~mm}$. La temporada de lluvias ocurre de julio a octubre y la de secas de noviembre a junio. La temperatura media anual es $20.8^{\circ} \mathrm{C}$, la mínima promedio de invierno $3.9^{\circ} \mathrm{C}$ y la máxima promedio en verano $36.1^{\circ} \mathrm{C}$ (Montaña y Breimer 1988; CONANP 2006).

Captura y monitoreo de venados bura. Las capturas se realizaron en los años 2011 y 2012 usando redes de caída de 15 x $15 \mathrm{~m}$ con una luz de 10 x $10 \mathrm{~cm}$. A las venadas capturadas se les colocaron radiocollares de colores distintos, con transmisores VHF (150 y $152 \mathrm{MHz}$ ) y sensor de movimiento (modelo 400, TELONICS, Mesa, Arizona), se monitorearon de enero de 2012 a agosto de 2014. Las localizaciones fueron registradas cada hora en ciclos continuos de 24 horas, se realizaron de dos a tres ciclos continuos por venada, aproximadamente cada dos meses y durante todo el periodo de estudio. Las lecturas se obtuvieron en forma

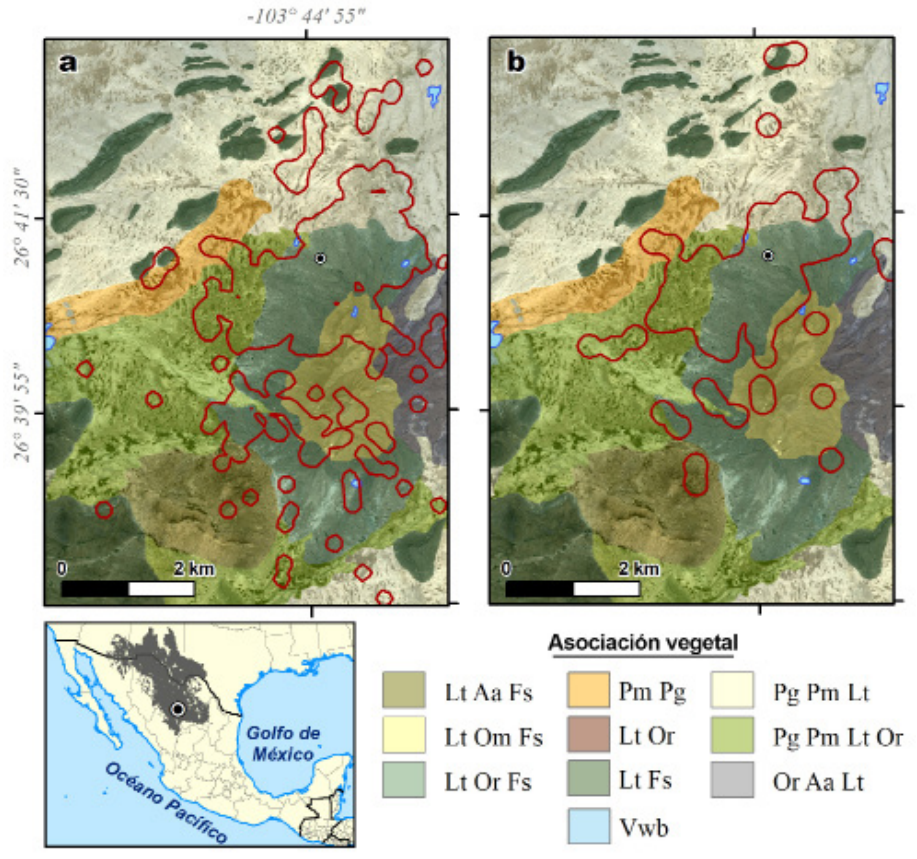

Figura 1. Áreas usadas por las hembras de venado bura para descansar (a) y alimentarse (b), así como las asociaciones vegetales presentes al interior de cada área. El Laboratorio del Desierto de Mapimí (circulo blanco y negro) se ubica al interior del Desierto Chihuahuense (polígono gris). Asociación vegetal: Pleuraphis mutica y Prosopis glandulosa (Pm Pg); Larrea tridentata y Fouquieria splendens (Lt Fs); Larrea tridentata, Agave asperrima y Fouquieria splendens (Lt Aa Fs); Larrea tridentata, Opuntia microdasys y Fouquieria splendens (Lt Om Fs); Larrea tridentata, Opuntia rastrera y Fouquieria splendens (Lt Or Fs); Opuntia rastrera, Agave asperrima y Larrea tridentata (Or Aa Lt); Prosopis glandulosa, Pleuraphis mutica y Larrea tridentata (Pg Pm Lt); Prosopis glandulosa, Pleuraphis mutica, Larrea tridentata y Opuntia rastrera (Pg Pm Lt Or) y vegetación asociada a cuerpos de agua ( $\mathrm{Vwb})$. 
simultánea en dos estaciones fijas, con antenas receptoras tipo "Yagui" ( 2 de 4 elementos) de pico nulo, ubicadas en sitios elevados. El error de estimación fue calculado usando radiocollares con una ubicación georeferenciada (White y Garrott 1990; Withey et al. 2001); el polígono de error de las localizaciones se consideró bajo el cálculo de extensión de $0.09 \mathrm{~km}^{2}$.

Pautas de comportamiento y patrón de actividad. Las pautas de comportamiento se determinaron a través del uso del sensor de movimiento que posee el radiotransmisor de los collares, el cual emite una señal o pulsación ("beeps") generada por una bola de mercurio ubicada en un tubo dentro del radiotransmisor. Al desplazarse el mercurio se producen cierres en un interruptor, lo que incrementa o disminuye el número de "beeps" emitidos por minuto. El movimiento de los animales al levantar o bajar la cabeza genera cambios en el número de "beeps" que se emiten, los cuales van de 50 a 87 dependiendo la posición de la cabeza, correspondiendo los valores más bajos cuando están echados y los más altos cuando están comiendo (Mandujano et al. 1996).

Durante cada localización hecha para cada venada, se contabilizó el número de "beeps" emitidos por minuto, y durante cinco minutos para minimizar el error de asignación de pauta. La asignación de la pauta correspondiente a la localización se hizo considerando la variación observada durante los cinco minutos de conteo (Mandujano et al. 1996). Se utilizaron los siguientes valores de beeps por minuto para identificar las tres pautas de comportamiento: descanso de 50 a 55 beeps/minuto, en movimiento (que incluye las posibles pautas de caminando, parado o alimentándose intermitentemente) de 56 a 69 beeps/minuto y alimentándose (casi exclusivamente en el mismo sitio) de 70 a 87 beeps/minuto. Esto se hizo siguiendo los resultados obtenidos con venados en cautiverio por Mandujano et al. (1996). Se caracterizó el patrón diario de actividad relacionado con estas tres pautas de comportamiento usando gráficas de densidad Kernel, con el paquete overlap (Ridout y Linkie 2009) en el programa R 3.6.0 (R Core Team 2019). Se consideró como actividad crepuscular de las 6:00 a 8:00 horas y de 18:00 a 20:00 horas, actividad diurna de 8:00 a 18:00 horas y actividad nocturna de 20:00 a 6:00 horas (Monroy-Vilchis et al. 2011).

Sitios de alimentación y descanso. Ya que los sitios de alimentación y descanso son importantes en la actividad de los individuos, por encontrarse en ellos algunos recursos críticos para sobrevivir, se evaluó cómo las pautas de alimentación y descanso eran afectadas por algunas variables del hábitat aplicando un modelo lineal generalizado (GLM, por sus siglas en inglés) con error tipo binomial en el programa R 3.6.0 (R Core Team 2019). Las localizaciones espaciales identificadas con estas pautas se relacionaron con las variables: pendiente del terreno, asociación vegetal presente, temperatura y humedad relativa. Las primeras dos variables se derivaron usando el sistema de información geográfica QGIS v.2.18 (QGIS Development Team 2017), donde la variable pendiente (grados) se extrajo de un modelo digital de elevación, con una resolución de 15 $\mathrm{m}$ por pixel. Las asociaciones vegetales fueron nombradas de acuerdo a las especies más dominantes (Montaña 1988), usando el mapa digital generado por Pérez-Solano et al. (2016), que identifica diez tipos de asociaciones vegetales (Figura 1). La temperatura y humedad relativa se obtuvieron cada hora durante los ciclos de muestreo mediante un termohigrómetro colocado en una de las dos estaciones de monitoreo (Extech RH101, Nashua, New Hampshire, USA). Consideramos que, dada la poca variación del terreno y de los sitios que ubicamos donde se movían las venadas, la temperatura y humedad no varía significativamente entre las localizaciones obtenidas por radiotelemetría y las estaciones de monitoreo.

Aunado a esto, se identificaron los sitios donde los individuos se alimentaban y descansaban, así como la extensión $\left(\mathrm{km}^{2}\right)$ de dichas áreas. Esto fue hecho estimando "la distribución del uso" ("utilization distribution", UD) con el método Kernel fijo, usado comúnmente para estimar los ámbitos hogareños. EI UD proporciona una densidad de probabilidad para reubicar al animal en cualquier lugar de acuerdo con las coordenadas $(X, Y)$ de su localización espacial (Calenge 2006). Se usó el $95 \%$ de las observaciones y un valor de suavizado (h) calculado mediante validación cruzada de mínimos cuadrados (LSCV), en el paquete AdehabitatHR (Calenge 2006) dentro del programa R 3.6.0 ( Core Team 2019). Los polígonos obtenidos de este análisis se usaron como referencia para clasificar en QGIS v.2.18 (QGIS Development Team 2017), los tipos de vegetación y el porcentaje que éstas ocupan dentro de las áreas que se identificaron que fueron usadas para alimentarse y como refugio para descanso.

\section{Resultados}

Siete hembras fueron capturadas en 95 días de esfuerzo, sin embargo, el sensor de actividad de un collar falló desde el inicio, por lo que el análisis se realizó con seis individuos. Se registraron localizaciones de las seis venadas durante un total de 328 días/venado. Durante los tres años de seguimiento se obtuvo una media \pm desviación estándar de $87.50 \pm 53.04$ registros por individuo para la pauta descanso, $29.33 \pm 21.81$ para en movimiento y $21.17 \pm 19.32$ para alimentándose.

Se encontró que las venadas dedican la mayor parte del tiempo a descansar (63.41\%), seguido de pautas como caminar, estar paradas o alimentándose intermitentemente (en movimiento, $21.26 \%$ ) y la menor cantidad de tiempo es dedicada a estar detenidas alimentándose (15.34\%). La alimentación y los movimientos de las hembras de venado bura son realizados principalmente en horas crepusculares, y presentan dos picos de actividad (Figura 2). De acuerdo con el GLM, la temperatura (devianza $=4.7 ; g . l .=1 ; P=0.02$ ) tuvo una relación positiva con las pautas de descanso y alimentándose. El área estimada para los sitios que los individuos seleccionan para descansar fue de $10.35 \mathrm{~km}^{2}$, mientras que las áreas donde se alimentan están restringidas a una 


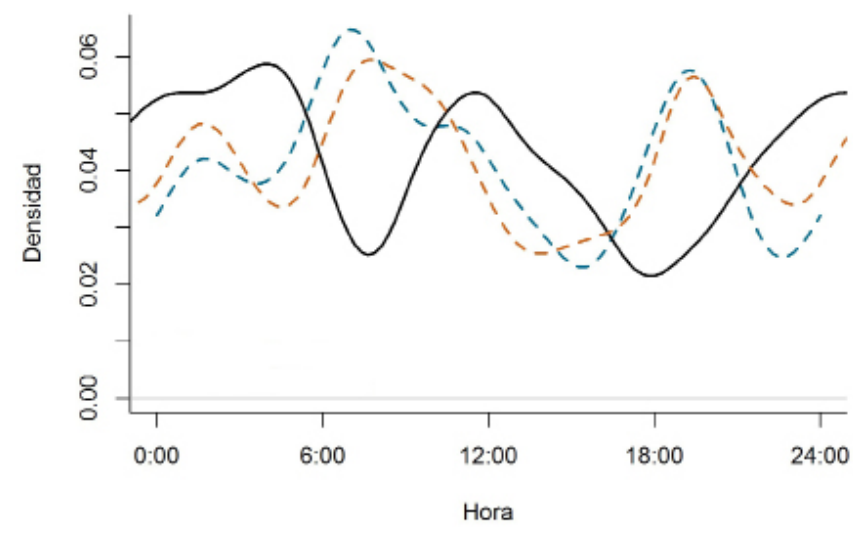

Figura 2. Patrón de actividad diario relacionado con tres pautas de comportamiento de seis hembras de venado bura al interior del Desierto Chihuahuense, México. La pauta descanso está representada por la línea negra, la pauta alimentación por la línea punteada marrón y la pauta movimiento por la línea punteada azul.

extensión de $6.45 \mathrm{~km}^{2}$ (Figura 1). Al interior de estas dos áreas se identificó la presencia de nueve de los diez tipos de asociaciones vegetales presentes en el área de estudio, la asociación de Larrea tridentata y Opuntia rastrera (Lt Or) estuvo ausente. La asociación vegetal con dominancia de Larrea tridentata, Opuntia rastrera y Fouquieria splendens ( $\mathrm{Lt}$ Or Fs) fue la que tuvo mayor presencia dentro de las áreas de alimentación y descanso (44 y $40 \%$ respectivamente; Figura 1 y 3), seguido de la asociación con Prosopis glandulosa, Pleuraphis mutica, L. tridentata y O. rastrera (Pg Pm Lt Or) también para ambos casos (22 y $20 \%$ respectivamente; Figura 1 y 3 ).

\section{Discusión}

En este estudio se encontró un patrón diario de actividad crepuscular para la especie, con picos de actividad al amanecer y anochecer, lo cual previamente ha sido reportado en trabajos en Estados Unidos (Relyea y Demarais 1994; Webb et al. 2013; Harris et al. 2015; Lendrum et al. 2017). En regiones desérticas los cérvidos, como el caso del venado bura, evaden las condiciones ambientales extremas a través del comportamiento (Wallmo 1981). De acuerdo con los resultados obtenidos, las venadas bura dedican la mayor proporción de tiempo a descansar (Figura 2). Considerando las altas temperaturas que predominan en el sitio así como la escases de agua en estas zonas áridas, el permanecer echadas la mayor parte del día les permite tener una adecuada termorregulación, al evitar la pérdida de agua por transpiración, y maximizar su energía (Gallina y Bello 2014), esto se refuerza con los resultados obtenidos en el GLM, donde la temperatura ambiental fue la única variable del hábitat que tuvo un efecto estadísticamente significativo sobre las pautas conductuales.

Por otra parte, las actividades de movimiento y alimentación tienen picos de actividad similar, sin embargo, el tiempo dedicado a alimentarse es menor del dedicado al desplazamiento (Figura 2). Al respecto, estudios con venado cola blanca han mostrado que los requerimientos diarios de energía disminuyen en latitudes más sureñas, en comparación con las norteñas, aunado a esto, en zonas

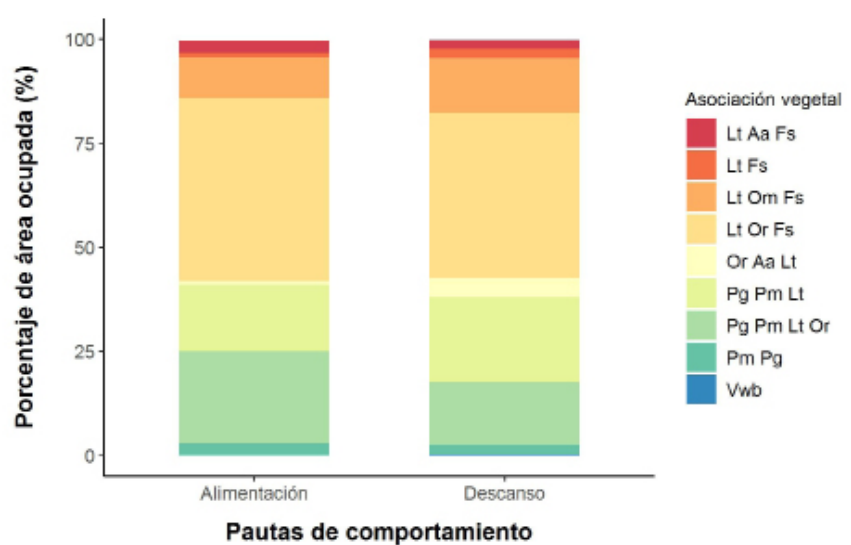

Figura 3. Porcentaje de área ocupada por las asociaciones vegetales presentes en las zonas de alimentación y descanso. Asociación vegetal: Pleuraphis mutica y Prosopis glandulosa (Pm Pg); Larrea tridentata y Fouquieria splendens (Lt Fs); Larrea tridentata, Agave asperrima y Fouquieria splendens (Lt Aa Fs); Larrea tridentata, Opuntia microdasys y Fouquieria splendens (Lt Om Fs); Larrea tridentata, Opuntia rastrera y Fouquieria splendens (Lt Or Fs); Opuntia rastrera, Agave asperrima y Larrea tridentata (Or Aa Lt); Prosopis glandulosa, Pleuraphis mutica y Larrea tridentata (Pg Pm Lt); Prosopis glandulosa, Pleuraphis mutica, Larrea tridentata y Opuntia rastrera (Pg Pm Lt Or) y vegetación asociada a cuerpos de agua (Vwb).

áridas la disponibilidad de recursos (agua y alimento) es limitada y los individuos se han adaptado a través de mecanismos tanto conductuales como fisiológicos (Strickland et al. 2005; Gallina y Bello 2010), esto ayudaría a explicar por qué las venadas en el área de estudio requieren dedicar menos tiempo a esta actividad. La necesidad de desplazarse está también relacionada con la distribución espacial de los recursos, y las venadas podrían estar invirtiendo más tiempo en esto, pues pueden estar desplazándose entre diversos parches de vegetación adecuada para alimentarse o para evadir a los depredadores (Esparza-Carlos et al. 2016).

La identificación de las áreas que las venadas usan para alimentarse y descansar indicó que están ocupando los mismos sitios (Figura 1), sin embargo, las áreas que usan para alimentarse son más pequeñas $\left(6.45 \mathrm{~km}^{2}\right)$ y se localizan al pie del Cerro San Ignacio, donde predomina la asociación vegetal dominada por Larrea tridentata, Opuntia rastrera y Fouquieria splendens, especies que han sido identificadas como parte esencial de la dieta del venado bura en la zona de estudio (Guth 1987; Gallina et al. 2017). Estas mismas áreas han sido previamente reportadas como las zonas de mayor uso por los venados por lo que son considerados como áreas núcleo de actividad (Pérez-Solano et al. 2017).

Finalmente, este es el primer trabajo que reporta el patrón de actividad para el venado bura en México con base en un método directo como la radiotelemetría, lo cual nos ayuda a entender un poco más sobre su ecología conductual y las estrategias adaptativas en ambientes áridos. Aún es necesario seguir estudiando otros aspectos biológicos y ecológicos de la especie, por ejemplo, la conducta de los machos, pues al ser una especie con una marcada segregación sexual los patrones conductuales, así como sus áreas de alimentación y refugio, probablemente son diferentes a las encontradas con las hembras en este estudio. De igual modo, sería importante considerar las etapas fisiológicas por las que pasan los individuos (por ejemplo, 
durante la temporada de apareamiento o gestación), para evaluar el efecto que tienen sobre la conducta de estos animales (Hungerford et al. 1981; Geist 1998).

\section{Agradecimientos}

Agradecemos al Consejo Nacional de Ciencia y Tecnología por el financiamiento al proyecto 132652 otorgado a SG-T, y por la beca de estudios 232620 otorgada a LAP-S. A The Rufford Foundation por la beca otorgada a LAP-S bajo el proyecto 15937-1. A la Comisión Nacional de Áreas Naturales Protegidas - Reserva de la Biosfera de Mapimí; a la Secretaría del Medio Ambiente y Recursos Naturales por los permisos otorgados (No. SEMARNAT/DGVS/00234 y 00954). A A. Sandoval-Comte por su apoyo en el uso de sistemas de información geográfica. A L. García-Feria, R. González-Trápaga y F. Herrera por su ayuda en campo, así como a los estudiantes que apoyaron en la captura y toma de datos.

\section{Literatura citada}

Alcalá-Galván, C. H., y P. Krausman. 2013. Home range and habitat use by desert mule deer in altered habitats. California Fish and Game 99:65-79.

Anderson, A. E., y O. C. Wallmo. 1984. Odocoileus hemionus. Mammalian Species 219:1-9.

Asensio, N., C. D. m Lusseau, M. Schaffner, y F. Aureli. 2012. Spider monkeys use high-quality core areas in a tropical dry forest. Journal of Zoology 287:250-258.

Avey, J. T., W. B. Ballard, M. C. Wallace, M. H. Humphrey, P. R. Krausman, F. Harwell, y E. B. Fish. 2003. Habitat relationships between sympatric mule deer and white-tailed deer in Texas. The Southwestern Naturalist 48:644-653.

BuRT, W. H. 1943. Territoriality and home range concepts as applied to mammals. Journal of Mammalogy 24:346-352.

CALENGE, C. 2006. The package "adehabitat" for the R software: A tool for the analysis of space and habitat use by animals. Ecological Modelling 197:516-519.

CONANP (Comisión Nacional de Áreas Naturales Protegidas). 2006. Programa de conservación y manejo Reserva de la Biosfera de Mapimí, México. Comisión Nacional de Áreas Naturales Protegidas. Distrito Federal, México.

Davies, N. B., J. R. Krebs, y S. A. West. 2012. An introduction to behavioural ecology. John Wiley and Sons. New York, U.S.A.

Esparza-Carlos, J. P., J. W. Laundré, L. Hernández, y L. I. ÍñiguezDÁvalos. 2016. Apprehension affecting foraging patterns and landscape use of mule deer in arid environments. Mammalian Biology 81:543-550.

Gallina, S., L. García, y R. González-Trápaga. 2017. Ocotillo flowers as food resource for the mule deer during the dry season. Therya 8:185-188.

Gallina, S., y J. Bello. 2002. Ecología conductual del venado cola blanca texano. Memorias VIII Simposio sobre venados en México “Ing. Jorge Villarreal González", Tlaxcala, México.

Gallina, S., y J. Bello. 2010. El gasto energético del venado cola blanca (Odocoileus virginianus texanus) en relación a la precipitación en una zona semiárida de México. Therya 1:9-22.

Gallina, S., y J. Bello. 2014. Patrones de actividad del venado cola blanca en el noreste de México. Therya 5:423-426.

GEIST, V. 1998. Deer of the World, their evolution, behavior and ecology. Stackpole books. Pennsylvania, U.S.A.

Guth, M. C. G. A. 1987. Hábitos alimenticios del venado bura (Odocoileus hemionus, Rafinesque 1817) en la Reserva de la Biosfera de Mapimí, Durango. Tesis de Licenciatura, ENEP-I UNAM. Distrito Federal, México.

Harris, G., J. G. Sanderson, J. Erz, S. E. Lehnen, y M. J, Butler. 2015. Weather and prey predict mammals' visitation to water. PLoS ONE 10:1-21.

Heffelfinger, J. R., C. Brewer, C. H. Alcalá-Galván, B. Hale, D. L. Weybright, B. F. Wakeling, L. H. Carpenter, y N. L. Dodd. 2006. Habitat Guidelines for Mule Deer: Southwest Deserts Ecoregion. Mule Deer Working Group, Western Association of Fish and Wildlife Agencies. Arizona, U.S.A.

Hungerford, C. R., M. D. Burke, y P. F. Ftolliot. 1981. Biology and population dynamics of mule deer in Southwestern United States. Pp. 109-131 in Deer biology, habitat requirements, and management in Western North America. (Ftolliot, P. F., y S. Gallina, eds.). Instituto de Ecología. Distrito Federal, México.

KaUfman, J. H. 1962. Ecology and social behavior of the coati Nasua narica on Barro Colorado Island, Panama. University of California publications in zoology 60:95-222.

Lendrum, P. E., K. R., Crooks, y G. Wittemyer. 2017. Changes in circadian activity patterns of a wildlife community post high-intensity energy development. Journal of Mammalogy 98:1265-1271.

Mandujano, S. 2004. Análisis bibliográfico de los estudios de venados en México. Acta Zoológica Mexicana (n. s.) 20:211-251.

Mandujano, S., S. Pérez, R. SÁnchez, y S. Gallina. 1996. Diferenciación de pautas de actividad del venado con ayuda de radiotransmisores con sensor de movimiento. Acta Zoológica Mexicana (n. s.) 67:67-78

MarChINTON, R., y D. HIRTH. 1984. Behavior. Pp. 129-168 in White-tailed deer, ecology and management (Halls, L. K., ed.) A Wildlife Management Institute Book. Stackpole books. Pennsylvania, U.S.A.

Marshal, J. P., V. C. Bleich, P. R. Krausman, M.L. Reed, y N. G. Andrew. 2006. Factors affecting habitat use and distribution of desert mule deer in an arid environment. Wildlife Society Bulletin 34:609-619.

Monroy-Vilchis, O., M. M. Zarco-González, C. Rodríguez-Soto, L. SORIA-DíAZ, y V. URIOS. 2011. Fototrampeo de mamíferos en la Sierra Nanchititla, México: abundancia relativa y patrón de actividad. Revista de Biología Tropical 59:373-383.

Montaña, C. 1988. Las formaciones vegetales. Pp. 167-197 in Estudio integrado de los recursos vegetación, suelo y agua en la reserva de la biosfera de Mapimí (Montaña, C. ed.). Instituto de Ecología. Distrito Federal, México.

Montaña, C., y R. F. Breimer. 1988. Major vegetation and environment units. Pp. 99-114 in Estudio integrado de los recursos vegetación, suelo y agua en la Reserva de la Biosfera de Mapimí (C. Montaña, ed.). Instituto de Ecología. Distrito Federal, México.

Morrison, M. L., B. Marcot, y W. Mannan. 2006. Wildlife-habitat relationships: concepts and applications. Island Press. Washington, U.S.A.

Pérez-Solano, L. A., S. Gallina-Tessaro, y G. Sánchez-Rojas. 2016. Individual variation in mule deer (Odocoileus hemionus) habitat and home range in the Chihuahuan Desert, Mexico. 
Journal of Mammalogy 97:1228-1237.

Pérez-Solano L. A., L. M. García-Feria, y S. Gallina-Tessaro. 2017. Factors affecting the selection of and displacement within core areas by female mule deer (Odocoileus hemionus) in the Chihuahuan Desert, Mexico. Mammalian Biology 87:152159.

PowelL, R. A. 2000. Animal home ranges and territories and home range estimators. Pp. 65-110 in Research techniques in animal ecology: controversies and consequences (Boitani, L., y T. K. Fuller, eds.) Columbia University Press. New York, U.S.A. QGIS Development Team. 2017. QGIS Geographic Information System. Open Source Geospatial Foundation Project. http:// qgis.osgeo.org.

R CORE TEAM. 2019. R: A language and environment for statistical computing. R Foundation for Statistical Computing, Vienna, Austria. https://www.R-project.org/.

Rzedowskı, J. 2006. Vegetación de México. Comisión Nacional para el Conocimiento y uso de la Biodiversidad. Distrito Federal, México.

Relyea, R.A., y S. Demarais. 1994. Activity of Desert Mule Deer during the Breeding Season. Journal of Mammalogy 75:940949.

RIDOUT, M., y M. LINKIE. 2009. Estimating overlap of daily activity patterns from camera trap data. Journal of Agricultural, Biological, and Environmental Statistics 14:322-337.

Samuel, M. D., y R. E. Green. 1988. A revised test procedure for identifying core areas within the home range. Journal of Animal Ecology 57:1067-1068.

SÁnchez-Rojas, G., y S. Gallina. 2000. Factors affecting habitat use by mule deer (Odocoileus hemionus) in the central part of the Chihuahuan Desert, Mexico: an assessment with univariate and multivariate methods. Ethology Ecology and Evolution 12:405-417.

Strickland, B. K., D. G. Hewitttt, C. A. Deyoung, y R. L. Bingham. 2005. Digestible energy requirements for maintenance of body mass of white-tailed deer in southern Texas. Journal of Mammalogy 86:56-60.

Wallmo, O. 1981. Mule and black-tailed deer of North America. A Wildlife Management Institute Book. University of Nebraska Press. Nebraska, U.S.A.

Webb, S. L., M. R. Dzialak, D. Houchen, K. L. Kosciuch, y J. B. WinSTEAD. 2013. Spatial ecology of female mule deer in an area proposed for wind energy development. Western North American Naturalist 73:347-356.

White, G. C., y R. A: Garrott. 1990. Analysis of radio-tracking data. Academic. San Diego, U.S.A.

Withey, J. C., T. D Bloxton, y J. M. MARZLUfF. 2001. Effects of tagging and location error in wildlife radiotelemetry studies. Pp. 4375 in Radio tracking and animal populations (Millspaugh, J. J., y J. M. Marzluff, eds.). Academic Press. San Diego, U.S.A. 